Vladas Vekteris

Andrius Trumpa

Vytautas Turla

Nikolaj Šešok

Igor Iljin

Vadim Mokšin

Artūras Kilikevičius

Arūnas Jakštas

Jonas Kleiza

DOI: 10.21278/TOF.41207

ISSN 1333-1124

eISSN 1849-1391

\title{
AN INVESTIGATION INTO FAULT DIAGNOSIS IN A ROTOR- BEARING SYSTEM WITH DAMPERS USED IN CENTRIFUGAL MILK SEPARATORS
}

\begin{abstract}
Summary
This paper deals with the problems encountered in the fault diagnosis in damped rotorbearing systems used in centrifugal milk separators. It is shown that direct bearing vibration measurements are more reliable than indirect (through rotor housing) vibration measurements. However, in most cases it is not possible to measure bearing vibrations directly. The factors causing large diagnostic measurement errors, such as the influence of the vibration damping system, step-up gear and electric motor noise are discussed in the paper. It is established that vibration measurements of the damping system are noninformative and cannot be used to diagnose faults in bearings. It is proposed to use the vibration spectra correlation to predict bearing failures in rotor-bearing systems of this type.
\end{abstract}

Key words: $\quad$ centrifugal milk separator, bearing fault diagnosis, vibration signal analysis, correlation.

\section{Introduction}

Rolling bearing faults can be categorized into inner race faults, outer race faults, rolling element faults, and cage faults. The rolling bearing fault is one of the main causes of failures in rotating machinery [1]. Such failures can lead to catastrophic consequences and costly downtime [2]. In order to prevent bearing faults, various bearing fault diagnosis techniques have been developed. The vibration signal analysis is the main bearing fault monitoring method [1].

In practice, the bearing fault detection is a difficult task [3]. It is known that vibration sources emit high levels of noise due to complex surroundings [1]. In most cases, defectinduced impulses are weak at the incipient stage, and often buried in the background noise [1, 
V. Vekteris, A. Trumpa, V. Turla,

N. Šešok, I. Iljin, V. Mokšin,

A. Kilikevičius, A. Jakštas, J. Kleiza
An Investigation Into Fault Diagnosis in a Rotor-Bearing System with Dampers Used in Centrifugal Milk Separators

3]. In such cases, the vibration-based methods tend to give a wrong diagnosis. It is therefore necessary to have a robust signal processing method for an effective extraction of incipient fault symptoms $[1,3,4]$.

Rolling bearing fault signals are usually transient and modulated by carrier signals of high frequency [5]. Some of the most useful signal processing approaches used for the bearing fault detection are enveloping (demodulation) methods, where the vibration signal is first processed by a classical high-pass filter to obtain a high signal-to-noise ratio signal [1]. Then, the Hilbert transform is applied to obtain the envelope. If periodic impulses excited by the bearing fault appear in the envelope corresponding to the bearing characteristic frequency, it can be stated that the bearing is under fault conditions [1]. In many cases, the enveloping methods are effective [1]. However, it is necessary to choose a proper cut-off frequency of the filter. This is a complicated task, especially if the noise type and frequency range are unknown [1].

Wavelet transform is another effective signal processing method used in the bearing fault diagnosis [6]. It has the local characteristic of time domain as well as frequency domain and its time-frequency window is changeable [1]. In comparison with traditional feature extraction methods it delivers better performance [7]. Prabhakar et al. established that the discrete wavelet transform can be effectively used to detect single and multiple bearing faults [8]. He et al. proposed a wavelet filter-based method that uses the filtering characteristic of the wavelet transform at a fixed scale [5]. Su et al. compared the performance of the discrete wavelet transform and the wavelet filter-based method used to detect faults in ball bearings [9]. The results were in favour of the wavelet filter-based method which was also adopted by the authors [1] to extract the fault characteristic signal (impulsive signal) from the noisy vibration signal. Although some progress has been made in this area, there exist many kinds of mother wavelets in signal processing, but not every wavelet is suitable for extracting the fault characteristic from the vibration signal [6].

A vibration signal model of a faulty rolling bearing was investigated in the study [1]. A bearing vibration system was considered as an under-damped second-order mass-spring-damper system. It was assumed that the rolling element hits the raceway and an impulse is produced when a fault occurs in the rolling element bearing. As the impulse with short time duration is a transient excitation, the resonance of the natural frequency of the bearing system arises.

It was shown in the study [10] that zero-crossing features extracted from time domain rotor vibrations are useful in the early detection and identification of bearing faults. The proposed model for fault detection does not require estimation of the rotational frequency of the rotor or specification of the geometry of the bearing or transformation of the time domain signal into another favourable spectral domain. With proper selection of the window size, the number of zero-crossing intervals and the interval thresholds, zero-crossing features combined with an artificial neural network are capable of high classification and low false alarm rates.

The majority of machine condition monitoring systems employ sophisticated algorithms to extract a representative set of features from the spectral domain [11] or the wavelet coefficient domain [12, 13]. In some cases the diagnosis is performed using statistical parameters [14], such as the root mean square, kurtosis, crest factor, etc. The remaining useful life of the machine is predicted using the vibration signal of a partially degrading bearing [15]. Many approaches propose combining time domain features with other features extracted from the frequency domain [11] to better approximate the vibration signature for a more accurate diagnosis. This explains the popularity of wavelet coefficients in the detection of bearing faults.

The magnitude of the vibration data can be used as an indication of a potential fault [10]. Early detection requires additional processing of the vibration data because some faults will not produce a significant change in the vibration data until a complete failure is about to 
happen. A single point bearing fault will produce a harmonic series with a fundamental frequency equal to one of four characteristic frequencies depending on which bearing surface the fault occurs. Assuming that the inner race is rotating with the shaft while the outer race is fixed, these four characteristic frequencies are given in Refs. [11, 16, 17].

This study demonstrates complexity of the bearing fault diagnosis process for rotorbearing systems equipped with vibration dampers that add considerable uncertainty to the results of diagnostic measurements. In such a case, additional studies are required to assess the reliability of the bearing fault diagnosis results.

\section{Mathematical study}

Separator housing vibrations are caused by the rotor unbalance, bearings, the electric motor and the gearbox. Equations of motion of the housing with a rotor, bearings and dampers can be written as follows:

$$
\begin{aligned}
& m_{1} \ddot{y}_{1}+h_{1}\left(\dot{y}_{1}-\dot{y}_{p}\right)+h_{2}\left(\dot{y}_{1}-\dot{y}_{2}\right)+c_{1}\left(y_{1}-y_{p}\right)+c_{2}\left(y_{1}-y_{2}\right)=0, \\
& m_{2} \ddot{y}_{2}+h_{2}\left(\dot{y}_{2}-\dot{y}_{1}\right)+h_{3}\left(\dot{y}_{2}-\dot{y}_{3}\right)+c_{2}\left(y_{2}-y_{1}\right)+c_{3}\left(y_{2}-y_{3}\right)=0, \\
& m_{3} \ddot{y}_{3}+h_{3}\left(\dot{y}_{3}-\dot{y}_{2}\right)+c_{3}\left(y_{3}-y_{2}\right)=0,
\end{aligned}
$$

where $m_{1}, m_{2}$ and $m_{3}$ are the masses of the rotor housing, rotor bearings with dampers and the bowl with a rotor, respectively (Fig. 1), $h_{i}, c_{i}$ are the damping and stiffness coefficients of the housing and bearings with dampers $(i=1,2,3)$ (Fig. 1), $y_{i}$ are the displacements of the housing, bearings and the rotor expressed in absolute coordinates $(i=1,2,3)$ (Fig. 1), $y_{p}$ is the occasional displacement of the base (Fig. 1).

Coefficient values in the system of equations (1) were obtained experimentally.

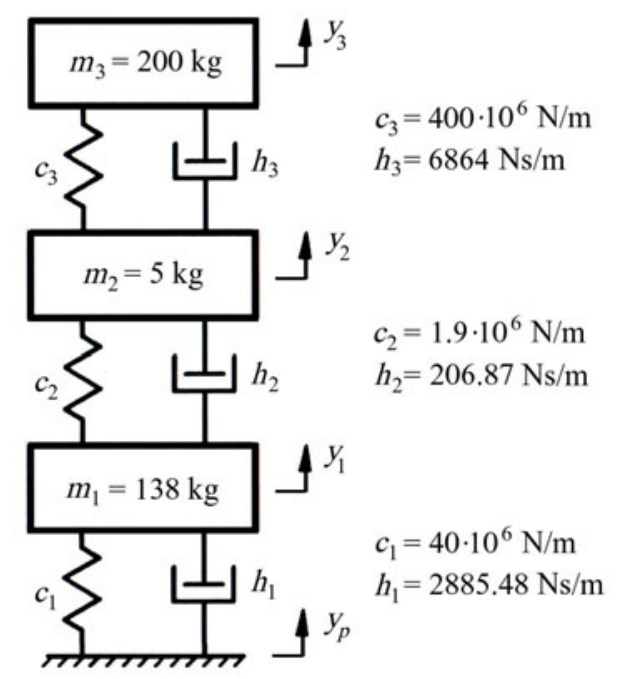

Fig. 1 Mass spring model of the rotor-bearing system of a separator

Using relative coordinates and considering $y_{p}=e^{j \omega t}$ and $y_{i}=H_{y_{i}}(j \omega) e^{j \omega t}$, after carrying out simple rearrangements according to [18], the system of equations (1) can be written in a matrix form as follows: 
V. Vekteris, A. Trumpa, V. Turla,

N. Šešok, I. Iljin, V. Mokšin,

A. Kilikevičius, A. Jakštas, J. Kleiza
An Investigation Into Fault Diagnosis in a Rotor-Bearing System with Dampers Used in Centrifugal Milk Separators

$$
\left[\begin{array}{c}
H_{y_{1}}(j \omega) \\
H_{y_{2}}(j \omega) \\
H_{y_{3}}(j \omega)
\end{array}\right]=\omega^{2}[z]^{-1}\left[\begin{array}{l}
1 \\
0 \\
0
\end{array}\right],
$$

where $\omega$ is the angular velocity of the rotor, $t$ is the time, $H_{y_{i}}(j \omega)$ is the complex transfer function $[19,20]$ and

$$
[z]=-\left[\begin{array}{lll}
1 & 0 & 0 \\
0 & 1 & 0 \\
0 & 0 & 1
\end{array}\right]+j \frac{1}{\omega}\left[\begin{array}{ccc}
\frac{h_{1}}{m_{1}} & -\frac{h_{2}}{m_{1}} & 0 \\
-\frac{h_{1}}{m_{1}} & \frac{h_{2}}{m_{1}}+\frac{h_{2}}{m_{2}} & -\frac{h_{3}}{m_{2}} \\
0 & -\frac{h_{2}}{m_{2}} & \frac{h_{3}}{m_{2}}+\frac{h_{3}}{m_{3}}
\end{array}\right]+\frac{1}{\omega^{2}}\left[\begin{array}{ccc}
\frac{c_{1}}{m_{1}} & -\frac{c_{2}}{m_{1}} & 0 \\
-\frac{c_{1}}{m_{1}} & \frac{c_{2}}{m_{1}}+\frac{c_{2}}{m_{2}} & -\frac{c_{3}}{m_{2}} \\
0 & -\frac{c_{2}}{m_{2}} & \frac{c_{3}}{m_{2}}+\frac{c_{3}}{m_{3}}
\end{array}\right] .
$$

Eq. 2 allows the calculation of the complex transfer function $H_{y_{i}}(j \omega)$. It allows one to analyse the excitation effect of the separator bowl on the vibration behaviour of the bearings and the housing. Graphical representation of Eq. 2 is presented in Fig. 2.

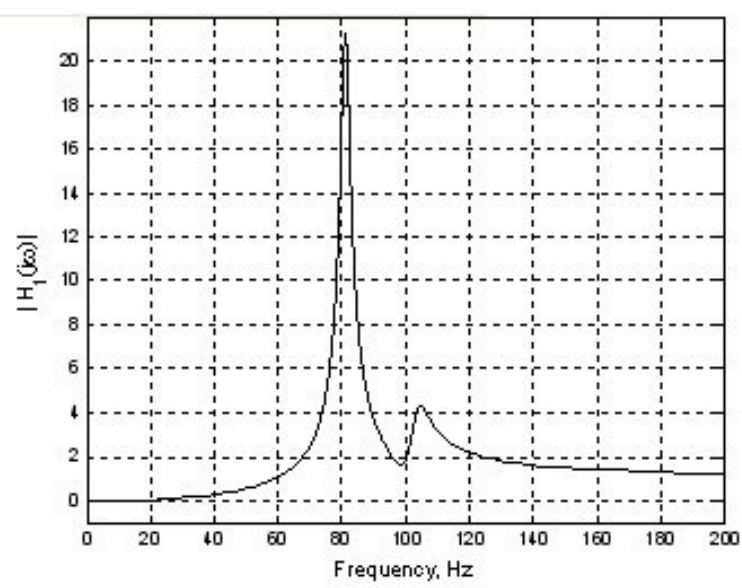

a

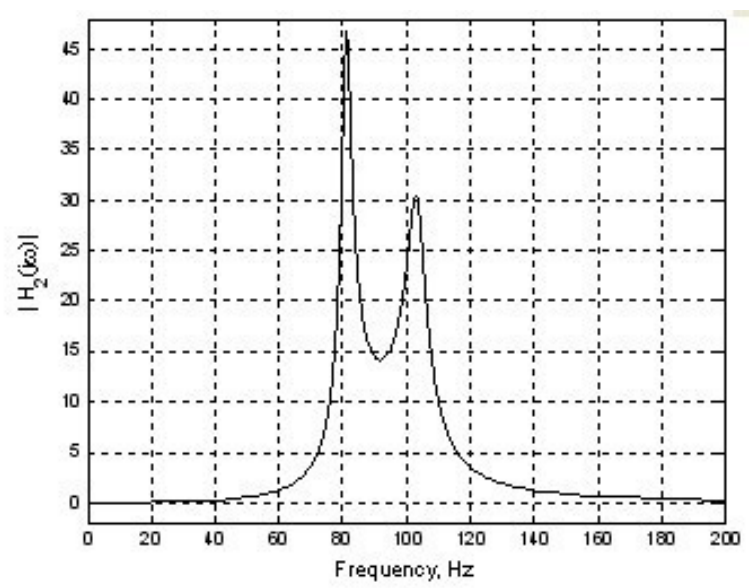

b

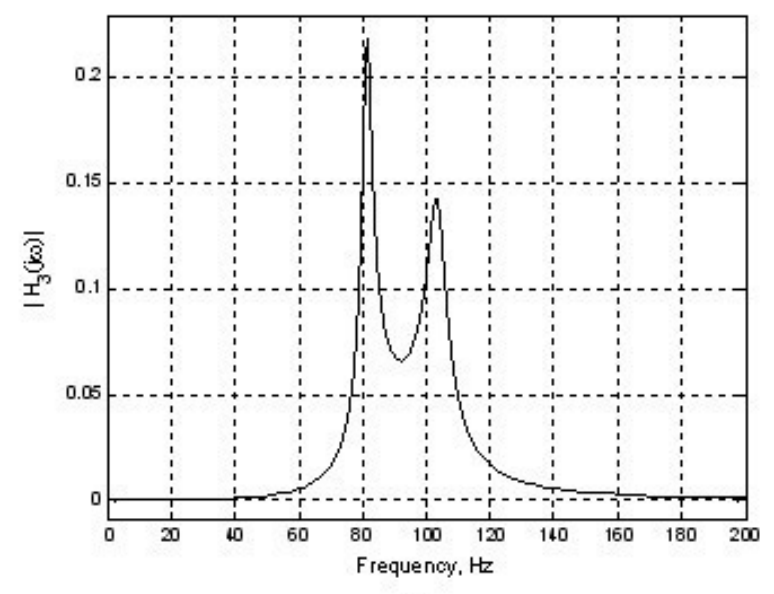

c

Fig. 2 Complex vibration transfer function plots: $a-$ transfer to the bearing, $b-$ transfer from the bearing to the damper, $\mathrm{c}-$ transfer from the damper to the housing 
An Investigation Into Fault Diagnosis in a Rotor-Bearing System with Dampers Used in Centrifugal Milk Separators
V. Vekteris, A. Trumpa, V. Turla, N. Šešok, I. Iljin, V. Mokšin, A. Kilikevičius, A. Jakštas, J. Kleiza

As it can be seen in Fig. 2, the vibration transfer to the bearing (Fig. 2a) is significant, as well as the vibration transfer from the bearing to the bearing damper (Fig. 2b). However, the transfer from the damper to the housing is low (Fig. 2c). This means that vibrations are well suppressed. In this way, the diagnostic measurements of vibrations of the centrifugal milk separator housing can be noninformative. However, this statement requires experimental verification.

\section{Experimental study}

Fig. 3 shows the centrifugal milk separator used in the study and Fig. 4 presents the vibration measurement scheme.

According to the diagnostic measurement scheme, vibration acceleration signals of the bearing and the housing were recorded in the cases when a faulty and a new, healthy bearing were used in the rotor-bearing system of the separator. Then, vibration velocity signals were obtained and spectral densities were calculated as well as the correlation functions, errors and uncertainties.

Obtained for the rotor rotational frequency of $112.4 \mathrm{~Hz}$, the vibration velocity frequency spectra are presented in Fig. 5.

It can be seen in Fig. 5a that the differences between the amplitudes of the faulty bearing and the healthy bearing are observed in the whole frequency range. If the vibrations of the housing are measured, these amplitude differences are observed at high frequencies only, i.e. from $1500 \mathrm{~Hz}$ to $6400 \mathrm{~Hz}$ (Fig. 5b).

The velocity amplitudes of the faulty bearing and the healthy bearing obtained in direct and indirect (through the housing) measurements differ by about ten times (Fig. 5).

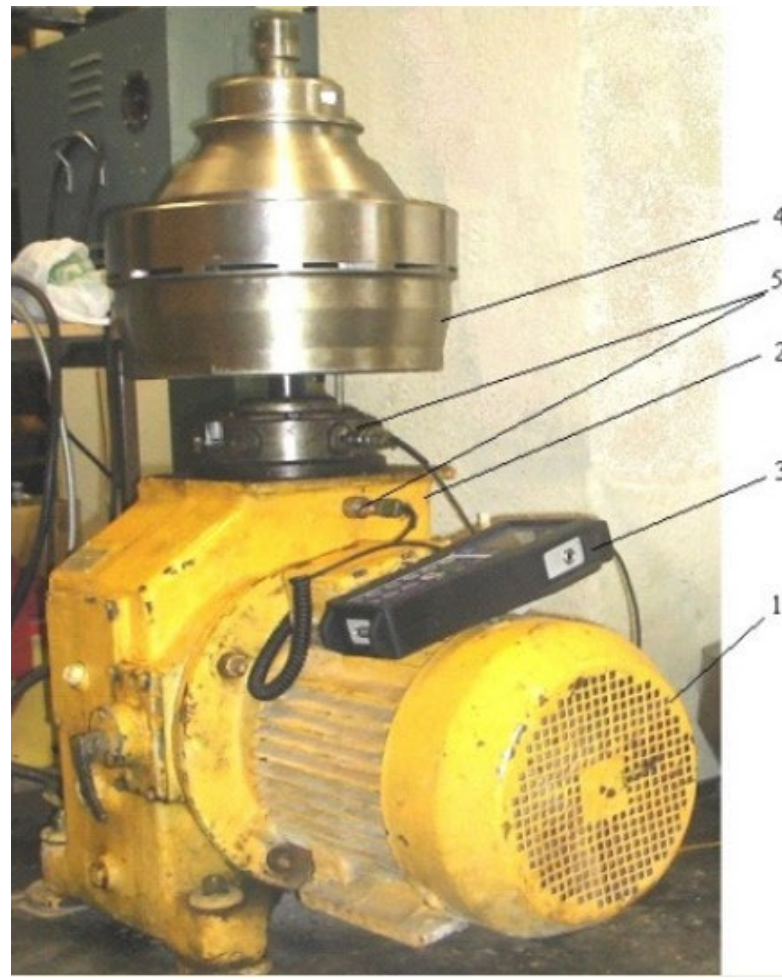

Fig. 3 Centrifugal milk separator (bowl shell is not shown): 1 - electric motor, 2 - step-up gear,

3 - computer, 4 - rotating bowl, 5 - accelerometer

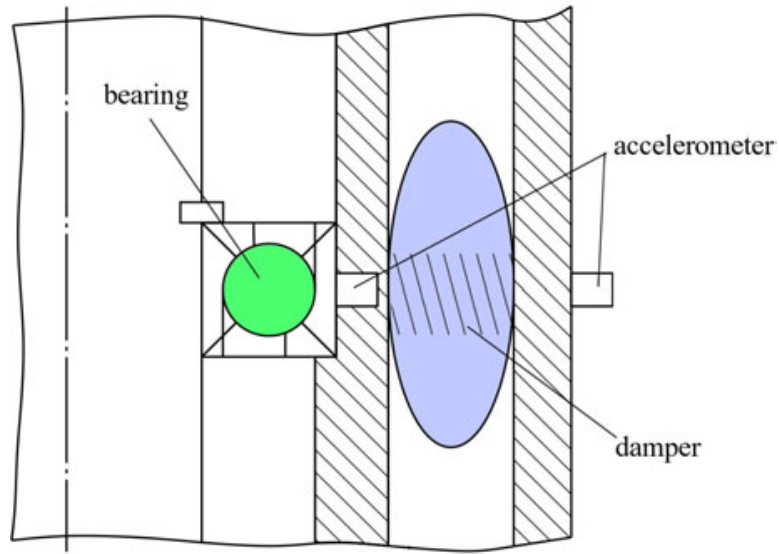

Fig. 4 Measurement scheme 
V. Vekteris, A. Trumpa, V. Turla,

N. Šešok, I. Iljin, V. Mokšin,

A. Kilikevičius, A. Jakštas, J. Kleiza

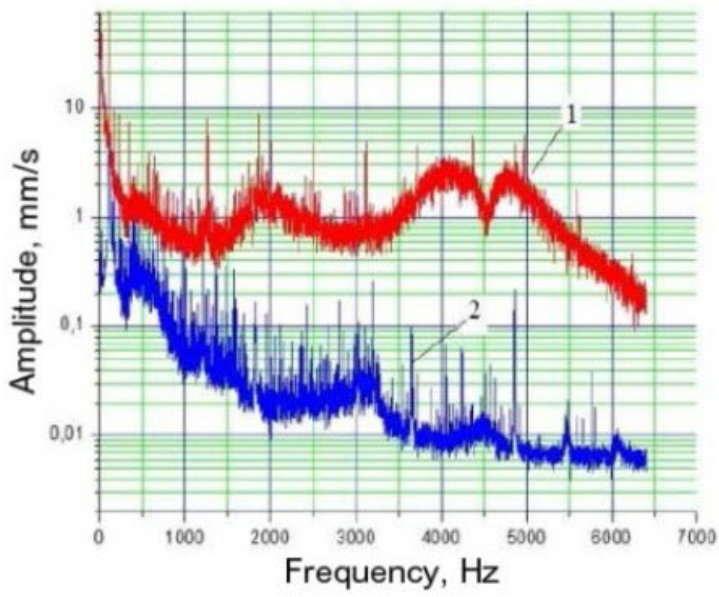

(a)
An Investigation Into Fault Diagnosis in a Rotor-Bearing System with Dampers Used in Centrifugal Milk Separators

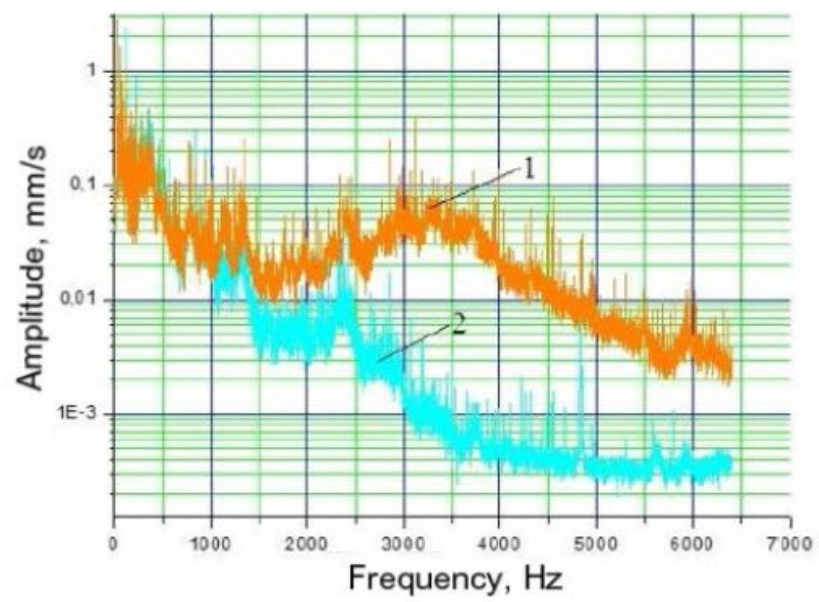

(b)

Fig. 5 Vibration velocity spectra: a - obtained from bearing vibration measurements, $b$ - obtained from housing vibration measurements, 1 - case when a faulty bearing was used in rotor-bearing system, 2 - case when a healthy bearing was used in rotor-bearing system

The vibration transfer functions presented in Fig. 6 show great influence of the vibration damping system.

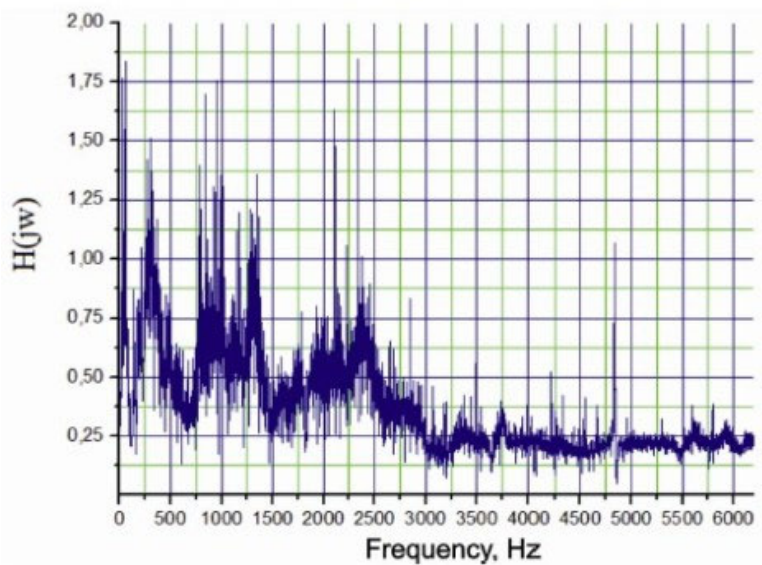

(a)

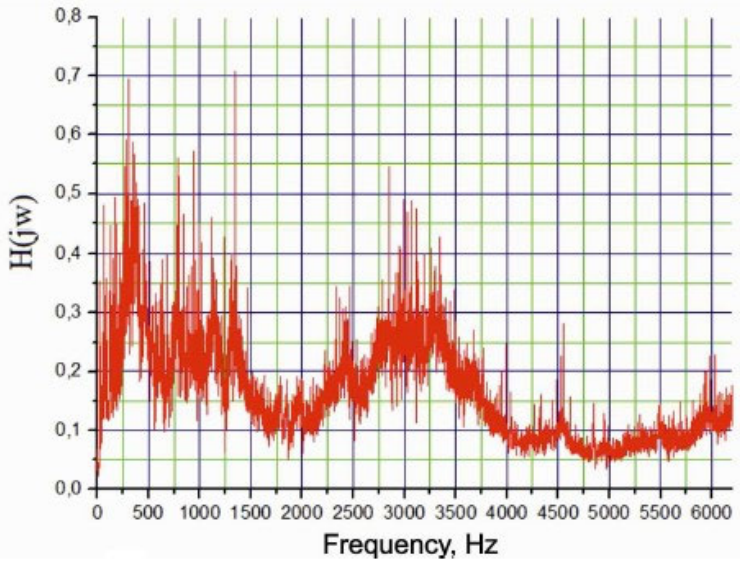

(b)

Fig. 6 Vibration transfer function plots: a - transfer from the healthy bearing to the housing, $\mathrm{b}$ - transfer from the faulty bearing to the housing

It can be stated that the vibration transfer from the bearing to the housing is different in the case when the faulty bearing was used and the case when the healthy bearing was used. Since the transfer function reflects the inertia of the system, some spectrum frequencies are amplified while others are suppressed. Thus, faulty bearing vibration frequencies ranging from 2000 to $4000 \mathrm{~Hz}$ are transmitted to the housing and the vibrations up to $2000 \mathrm{~Hz}$ do not show significant differences as compared with the healthy bearing (Fig. 6).

The results of vibration measurements of the damping system are presented in Fig. 7. High frequency vibrations (from 1500 to $6400 \mathrm{~Hz}$ ) of the faulty bearing dominate in the damping system spectra. Thus, the vibration measurements through the damping system are low informative and reliable, especially at frequencies up to $1500 \mathrm{~Hz}$, i.e., at the real frequencies of the bearing. 
An Investigation Into Fault Diagnosis in a Rotor-Bearing System with Dampers Used in Centrifugal Milk Separators
V. Vekteris, A. Trumpa, V. Turla, N. Šešok, I. Iljin, V. Mokšin, A. Kilikevičius, A. Jakštas, J. Kleiza

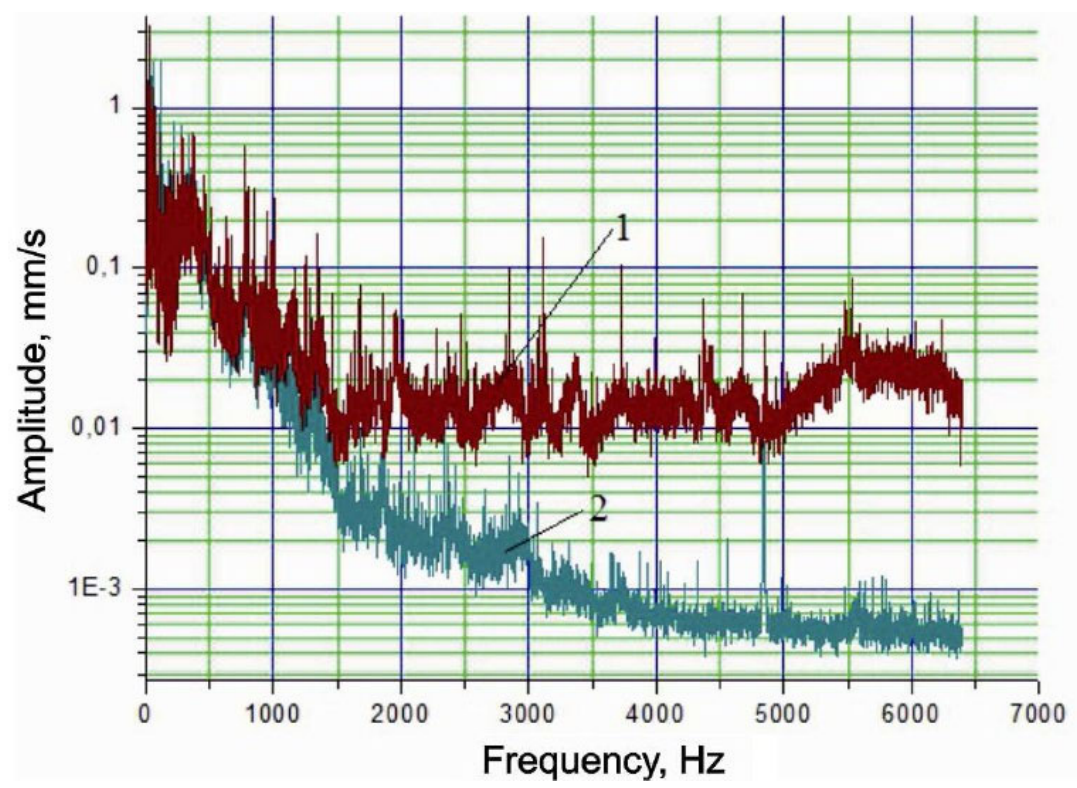

Fig. 7 Vibration velocity spectra of the damping system: 1 - case when the faulty bearing was used in rotorbearing system, 2 - case when the healthy bearing was used in rotor-bearing system

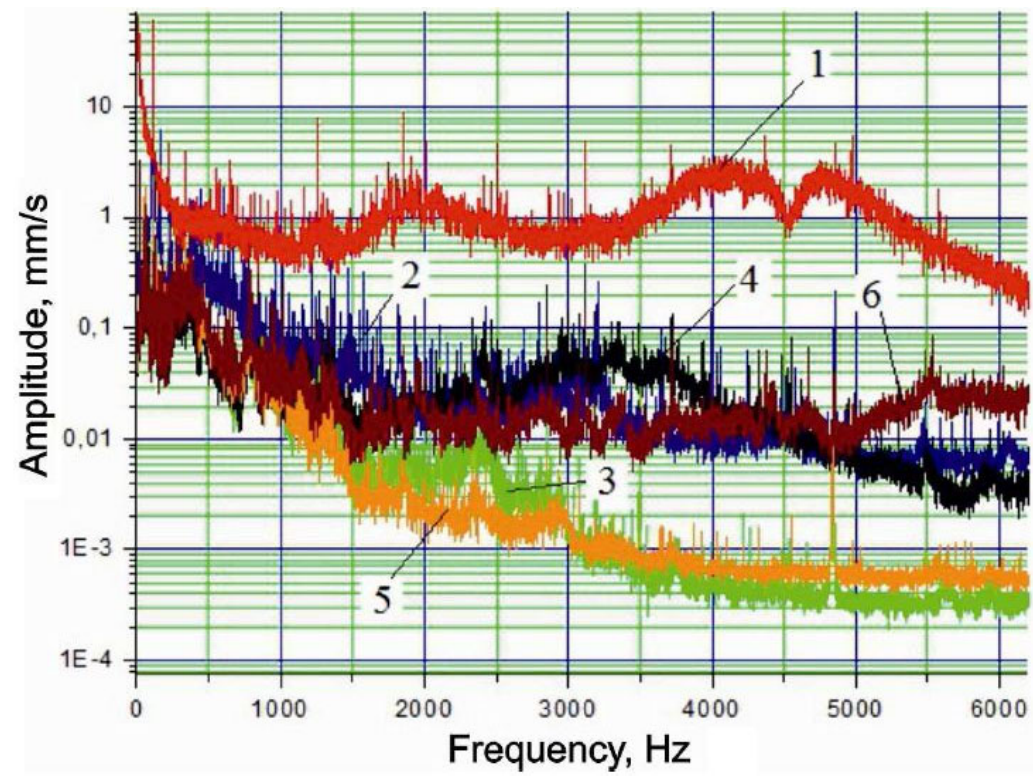

Fig. 8 Vibration velocity spectra: 1 - of the faulty bearing, 2 - of the healthy bearing, 3 - of the housing when the healthy bearing was used, 4 - of the housing when the faulty bearing was used, 5 - of the damper when the healthy bearing was used, 6 - of the damper when the faulty bearing was used

The vibration velocity spectra obtained by means of four accelerometers are presented in Fig. 8. The vibration amplitudes of the damping system do not differ in overall spectra in the case when the faulty bearing is used in the rotor-bearing system of the separator. Thus, by measuring the vibrations of the bearing housing, a decision about the bearing fault can be made only by employing high-frequency amplitudes (Fig. 7), i.e. starting from $1500 \mathrm{~Hz}$. However, this diagnostic information may be affected by vibrations of the other installed mechanisms.

It can be concluded that indirect diagnostic vibration measurements show significantly reduced amplitudes at frequencies up to $1500 \mathrm{~Hz}$.

As the housing vibration measurement results differ from the direct bearing vibration measurement results, the reliability of the fault diagnosis can only be assessed by using the 
V. Vekteris, A. Trumpa, V. Turla,

N. Šešok, I. Iljin, V. Mokšin,

A. Kilikevičius, A. Jakštas, J. Kleiza
An Investigation Into Fault Diagnosis in a Rotor-Bearing System with Dampers Used in Centrifugal Milk Separators

correlation function calculated for the respective frequencies. The correlation between the healthy bearing velocity spectrum and the faulty bearing velocity spectrum (Fig. 5a) is weak and positive according to the obtained correlation coefficient $(r=0.34168)$. The correlation between the housing velocity spectra (Fig. 5b) obtained for the healthy and the faulty bearing is strong and positive according to the correlation coefficient $(r=0.85954)$. This indicates that the damping system greatly suppresses the faulty bearing vibrations and the housing amplitudes do not depend on the condition of the bearing.

Therefore, based on these results, it is not possible to make an unambiguous decision about the suitability of the bearings. Further analysis of the correlation functions and the correlation coefficients is required. For this purpose it is necessary to establish a correlation between the bearing and the housing vibration spectra obtained in the case when the rotor is supported by a healthy bearing as well as a correlation between the bearing and the housing vibration spectra obtained in the case when the rotor is supported by a faulty bearing. The calculation results obtained by using the Microcal Origin ${ }^{\circledR} 7.0$ software are presented in Fig. 9.

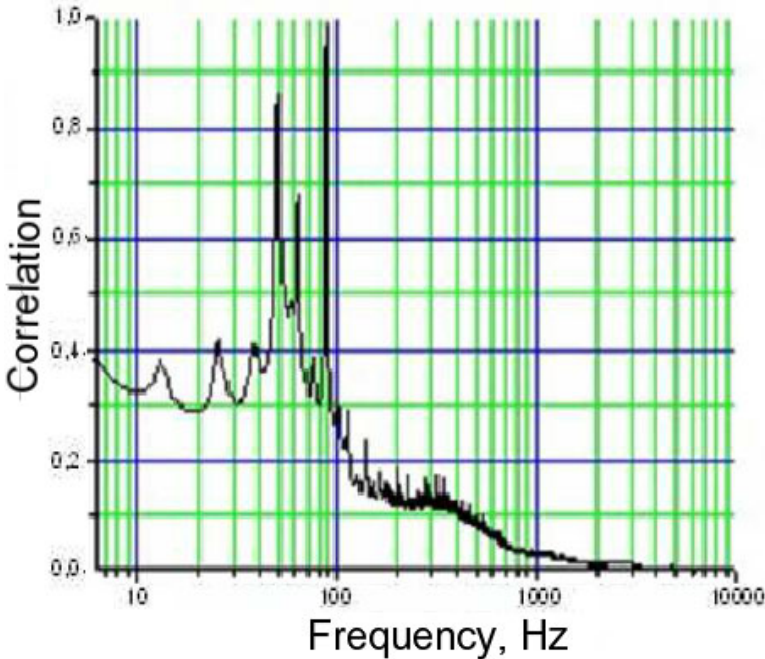

(a)

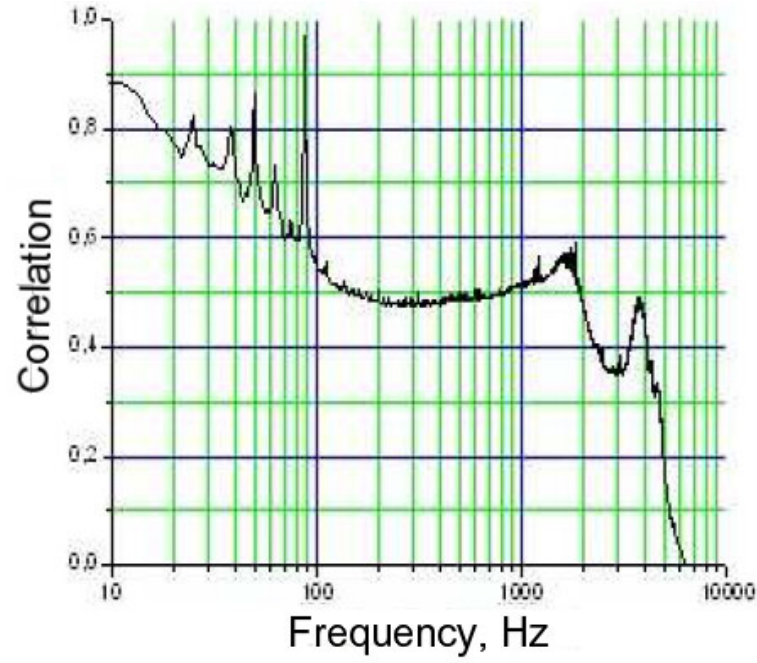

(b)

Fig. 9 Correlation function between vibration spectra of bearing and housing: a - obtained for the case when a healthy bearing was used, $\mathrm{b}$ - obtained for the case when a faulty bearing was used

The results obtained show that the correlation between the bearing and the housing vibration spectra in the case when the rotor is supported by the healthy bearing exists only at the bearing cage frequency and at the rotor rotational frequency. At other frequencies, the correlation is weak (Fig. 9a) and the total correlation coefficient reaches 0.6158 , which shows that the correlation is average. The correlation between the faulty bearing vibration spectrum and the housing vibration spectrum in the case when the rotor is supported by the faulty bearing occurs only at the bearing cage frequency and the rotor rotational frequency as well as at high frequencies (Fig. 9b). The total correlation coefficient $(r=0.2714)$ shows a very weak correlation.

Thus, these results demonstrate a range of problems encountered during the use of indirect measurements to diagnose faults in bearings. The combined standard uncertainty can be calculated according to the following formula:

$$
\left.u_{c}^{2} \approx \sum_{i=1}^{n} \frac{\partial f}{\partial x_{i}}\right|_{x_{i}=\bar{x}_{i}} ^{2} u_{x_{i}}^{2}+\left.2 \sum_{i=1}^{n-1} \sum_{j=i+1}^{n} \frac{\partial f}{\partial x_{i}} \frac{\partial f}{\partial x_{j}}\right|_{x_{i, j}-\bar{x}_{i, j}} u\left(x_{i}, x_{j}\right) .
$$

where the measured quantities $x_{i}$ are related by a functional relationship $f$. 
An Investigation Into Fault Diagnosis in a Rotor-Bearing System with Dampers Used in Centrifugal Milk Separators
V. Vekteris, A. Trumpa, V. Turla, N. Šešok, I. Iljin, V. Mokšin, A. Kilikevičius, A. Jakštas, J. Kleiza

According to Eq. 4 the value of $1.571 \mathrm{~mm} / \mathrm{s}$ was obtained. This is a very high value that influences the reliability of the obtained results.

It is known from the diagnostic measurements that the bearing outer ring vibration signal $X_{i}$ and the housing vibration signal $X_{j}$ are correlated, i.e. they are interdependent to some degree $-x_{i}$ and $x_{j}$ covariance, which provides an additional contribution to the uncertainty and is calculated by the following formula:

$$
u\left(x_{i}, x_{j}\right)=u\left(x_{i}\right) u\left(x_{j}\right) r\left(x_{i}, x_{j}\right),
$$

where $r\left(x_{i}, x_{j}\right)$ is the correlation coefficient $\left(-1<r\left(x_{i}, x_{j}\right)<1\right)$.

The relative error calculated from the vibration spectra obtained for the healthy bearing and the housing ranges from $5 \%$ to $1 \%$ at low and medium frequencies, while the relative error calculated from the vibration spectra obtained for the faulty bearing and the housing ranges from $15 \%$ at low and medium frequencies to $3 \%$ at the high frequencies.

Therefore, the results of the indirect diagnostic measurements can be incorrectly interpreted. This requires consideration of the diagnostic method selection and the realization aspects. The vibration spectra correlation analysis may be suitable for the prediction of bearing failures only.

\section{Conclusions}

1. Differences in the vibration velocity amplitude of the healthy and the faulty bearing occur only in the case of direct diagnostic measurements. The results of the indirect diagnostic measurements show that the fault diagnosis may be insufficient at frequencies higher than $1500 \mathrm{~Hz}$. The results of the measurements obtained in the case when the accelerometer is attached to the damping system are little informative and cannot be used to diagnose the state of the bearing.

2. After the detailed analysis of the vibration velocity spectra and their correlations, it is proposed to assess the reliability of the diagnostic measurements by applying the correlation relationship between the vibration spectra obtained from the direct and the indirect measurements. The correlation between the healthy bearing and the housing vibration spectra exists only at the bearing cage frequency and at the rotor rotational frequency. At other frequencies, the correlation is weak and the total correlation coefficient reaches 0.6158 , which shows that the correlation is average. The correlation between the faulty bearing vibration spectrum and the housing vibration spectrum occurs only at the bearing cage frequency and the rotor rotational frequency as well as at high frequencies. The total correlation coefficient $(r=0.2714)$ shows a very weak correlation.

3. The correlation relationship between the vibration spectra obtained from the indirect measurements can be insufficient for bearing failure prediction.

\section{REFERENCES}

[1] K. Feng, Z. Jiang, W. He and Q. Qin: Rolling element bearing fault detection based on optimal antisymmetric real Laplace wavelet, Measurement, 44, 9, 2011, 1582-1591, DOI:10.1016/j.measurement.2011.06.011

[2] J. Furch, J. Glos and J. Blecha: Identifying the technical condition of rotating parts by means of vibrodiagnostics, T Famena, 39, 2, 2015, 75-86

[3] Z. Li, Z. He, Y. Zi and Y. Wang: Customized wavelet denoising using intra- and inter-scale dependency for bearing fault detection, J Sound Vib, 313, 1-2, 2008, 342-359, DOI:10.1016/j.jsv.2007.11.039

[4] Z.-L. Lv, B.-P. Tang, Y. Zhou and C.-D. Zhou: A novel fault diagnosis method for rotating machinery based on EEMD and MCKD, Int J Simul Model, 14, 3, 2015, 438-449. DOI:10.2507/IJSIMM14(3)6.298

[5] W. He, Z.N. Jiang and K. Feng: Bearing fault detection based on optimal wavelet filter and sparse code shrinkage, Measurement, 42, 7, 2009, 1092-1102, DOI:10.1016/j.measurement.2009.04.001 
V. Vekteris, A. Trumpa, V. Turla,

N. Šešok, I. Iljin, V. Mokšin,

A. Kilikevičius, A. Jakštas, J. Kleiza
An Investigation Into Fault Diagnosis in a Rotor-Bearing System with Dampers Used in Centrifugal Milk Separators

[6] Z.K. Peng and F.L. Chu: Application of the wavelet transform in machine condition monitoring and fault diagnostics: A review with bibliography, Mech Syst Signal Pr, 18, 2, 2004, 199-221, DOI:10.1016/s0888-3270(03)00075-X

[7] X. Lou and K.A. Loparo: Bearing fault diagnosis based on wavelet transform and fuzzy inference, Mech Syst Signal Pr, 18, 5, 2004, 1077-1095, DOI:10.1016/s0888-3270(03)00077-3

[8] S. Prabhakar, A.R. Mohanty and A.S. Sekhar: Application of discrete wavelet transform for detection of ball bearing race faults, Tribol Int, 35, 12, 2002, 793-800, DOI:10.1016/s0301-679x(02)00063-4

[9] W. Su, F. Wang, H. Zhu, Z. Zhang and Z. Guo: Rolling element bearing faults diagnosis based on optimal Morlet wavelet filter and autocorrelation enhancement, Mech Syst Signal Pr, 24, 5, 2010, 1458-1472, DOI:10.1016/j.ymssp.2009.11.011

[10] P.E. William and M.W. Hoffman: Identification of bearing faults using time domain zero-crossings, Mech Syst Signal Pr, 25, 8, 2011, 3078-3088, DOI:10.1016/j.ymssp.2011.06.001

[11] B. Li, M.Y. Chow, Y. Tipsuwan and J.C. Hung: Neural-network-based motor rolling bearing fault diagnosis, IEEE T Ind Electron, 47, 5, 2000, 1060-1069, DOI:10.1109/41.873214

[12] J. Altmann and J. Mathew: Multiple band-pass autoregressive demodulation for rolling-element bearing fault diagnosis, Mech Syst Signal Pr, 15, 5, 2001, 963-977, DOI:10.1006/mssp.2001.1410

[13] S. Abbasion, A. Rafsanjani, A. Farshidianfar and N. Irani: Rolling element bearings multi-fault classification based on the wavelet denoising and support vector machine, Mech Syst Signal Pr, 21, 7, 2007, 2933-2945, DOI:10.1016/j.ymssp.2007.02.003

[14] F. Immovilli, M. Cocconcelli, A. Bellini and R. Rubini: Detection of generalized-roughness bearing fault by spectral-kurtosis energy of vibration or current signals, IEEE T Ind Electron, 56, 11, 2009, 4710-4717, DOI:10.1109/tie.2009.2025288

[15] N. Gebraeel, M. Lawley, R. Liu and V. Parmeshwaran: Residual life predictions from vibration-based degradation signals: A neural network approach, IEEE T Ind Electron, 51, 3, 2004, 694-700, DOI:10.1109/tie.2004.824875

[16] J.R. Stack, R.G. Harley and T.G. Habetler: An amplitude modulation detector for fault diagnosis in rolling element bearings, IEEE T Ind Electron, 51, 5, 2004, 1097-1102, DOI:10.1109/iecon.2002.1182940

[17] H. Ocak, K.A. Loparo: Estimation of the running speed and bearing defect frequencies of an induction motor from vibration data, Mech Syst Signal Pr, 18, 3, 2004, 515-533, DOI:10.1016/s0888-3270(03)00052-9

[18] J. M. Tessarzik, T. Chiang and R. H. Badgley: Effects on Vibration and Shocks on the Performance of Gas-Bearing Space-Power Brayton Cycle Turbomachinery. Part 3: Sinusoidal and Random Vibration Data Reduction and Evaluation, and Random Vibration Probability Analysis. Report NASA CR-121178, Mechanical Technology Incorporated, Latham, New York, 1973.

[19] R. Žigulić: Application of the complex transfer functions in robotics, Strojarstvo, 42, 5-6, 2000, $243-248$.

[20] M. Jokić, M. Stegić and N. Kranjčević: Structural stiffness optimization with respect to vibration response, T Famena, 36, 2, 2012, 1-8.

Submitted: $\quad 10.3 .2016$

Accepted: $\quad 20.9 .2016$ prof. Vladas Vekteris, Dr. Habil. lect. Andrius Trumpa, $\mathrm{Ph}$. D. prof. Vytautas Turla, Ph. D. assoc. prof. Nikolaj Šešok, Ph. D. assoc. prof. Igor Iljin, Ph. D. prof. Vadim Mokšin ${ }^{*}, \mathrm{Ph}$. D. prof. Artūras Kilikevičius, Ph. D. prof. Arūnas Jakštas, Ph. D.

Vilnius Gediminas Technical University

Faculty of Mechanics

J. Basanavičiaus str. 28

03224 Vilnius, Lithuania prof. Jonas Kleiza, Ph. D.

Vilnius Gediminas Technical University Faculty of Fundamental Sciences Saulètekio ave. 11 10223 Vilnius, Lithuania *corresponding author, vadim.moksin@vgtu.lt 